\title{
A constructive test for exponential stability of linear time-varying discrete-time systems
}

\author{
Ulrich Oberst ${ }^{1}$
}

Received: 13 January 2016 / Revised: 5 December 2016 / Accepted: 3 February 2017 / Published online: 15 February 2017

(C) The Author(s) 2017. This article is published with open access at Springerlink.com

\begin{abstract}
We complete the stability results of the paper Bourlès et al. (SIAM J Control Optim 53:2725-2761, 2015), and for this purpose use the linear time-varying (LTV) discrete-time behaviors and the exponential stability (e.s.) of this paper. In the main theorem we characterize the e.s. of an autonomous LTV system by standard spectral properties of a complex matrix connected with the system. We extend the theory of discrete-time LTV behaviors, developed in the quoted publication, from the coefficient field of rational functions to that of locally convergent Laurent series or even of Puiseux series. The stability test can and has to be applied in connection with the construction of stabilizing compensators.
\end{abstract}

Keywords Exponential stability · Discrete-time behavior $\cdot$ Linear time-varying

Mathematics Subject Classification 93D20 - 93C55 - 93C05

\section{Introduction}

We complete the stability results of [4] and use the notions, in particular the linear timevarying (LTV) discrete-time behaviors and the exponential stability (e.s.), of this paper, but extend the theory from the coefficient field $\mathbb{C}(z)$ of rational functions to the larger field $\mathbb{C}<<z>>$ of locally convergent Laurent series with at most a pole at 0 . In the main Theorem 1.1 together with Corollary 3.11 we characterize e.s. of an autonomous LTV system by standard spectral properties of a complex matrix connected with the system. Due to, for instance, $[6,14]$, Theorem 1.1 furnishes a constructive test for e.s.

Ulrich Oberst

ulrich.oberst@uibk.ac.at

1 Institut für Mathematik, Universität Innsbruck, Technikerstrasse 13, 6020 Innsbruck, Austria 
This test can and has to be applied in connection with the construction of stabilizing compensators; cf. [12] in the case of differential LTV systems.

The proof of Theorem 1.1 on state space behaviors is contained in Sect. 2. Section 3 presents the theory of discrete-time LTV behaviors for the coefficient field $\mathbb{C}<<z>>$, but we expose the necessary modifications of [4] only. In particular, behaviors and their morphisms, autonomous behaviors and their e.s. are recalled from [4] and adapted. Essential properties of these are stated in Corollary 3.10 on modulebehavior duality, in Corollary 3.12 on closure properties of the class of e.s. autonomous behaviors and in Corollary 3.11. The latter enables the application of Theorem 1.1 to arbitrary autonomous behaviors instead of state space behaviors only.

In Sect. 4 we shortly extend the results to the still larger field of locally convergent Puiseux series (cf. [16], [5, §3.1]). The latter field seems to be the largest coefficient field for which a reasonable stability theory for general LTV systems can be developed. We refer to the books [15, pp. 423-461] and [8, pp. 193-368] for comprehensive surveys of exponential stability of state space systems. Part II of the book [3] contains a detailed theory of general LTV behaviors and their stability that was modified in the papers $[4,5]$. We also refer to the recent papers $[1,2,7,10,13]$.

Theorem 1.1 requires some preliminary explanations: let $\mathbb{C}<z>$ denote the local principal ideal domain of locally convergent power series in the variable $z$ and $\mathbf{K}=$ : $\mathbb{C}<<z>>$ its quotient field. A formal power series $a=\sum_{i=0}^{\infty} a_{i} z^{i}$ is called locally convergent if

$$
\sigma(a):=\limsup _{i \in \mathbb{N}} \sqrt[i]{\left|a_{i}\right|}<\infty \text {. Then } \rho(a):=\sigma(a)^{-1}
$$

is its convergence radius and $a(z)$ is holomorphic in the open disc

$$
\mathbf{D}(\rho):=\{z \in \mathbb{C} ;|z|<\rho\}, \quad \rho:=\rho(a) .
$$

The power series $a$ is a unit (invertible) if and only if $a(0)=a_{0} \neq 0$, and $z$ is the unique prime of $\mathbb{C}<z>$, up to units. Each nonzero $a \in \mathbb{C}<z>$ has a unique representation

$$
a=z^{k} b, k:=\operatorname{ord}(a):=\min \left\{i ; a_{i} \neq 0\right\} \in \mathbb{N}, b=\sum_{j=0}^{\infty} a_{j+k} z^{j} \text { invertible. }
$$

This implies that every nonzero $a \in \mathbf{K}$ has the analogous unique representation

$$
\begin{aligned}
a(z) & =z^{k} b=\sum_{j=k}^{\infty} b_{j-k} z^{j}, \operatorname{ord}(a):=k \in \mathbb{Z}, b:=\sum_{i=0}^{\infty} b_{i} z^{i}, b_{0}=b(0) \neq 0 \\
\sigma(a) & :=\sigma(b):=\limsup _{i \in \mathbb{N}} \sqrt[i]{\left|b_{i}\right|}<\infty, \rho(a):=\sigma(a)^{-1} .
\end{aligned}
$$

The element $a$ is a power series if and only if $k=\operatorname{ord}(a) \geq 0$. The representation $a=\sum_{j=k}^{\infty} b_{j-k} z^{j}$ shows that $a$ is a locally convergent Laurent series with at most 
a pole at zero, and that indeed $\mathbf{K}=\mathbb{C}<<z>>$ consists of all these Laurent series. If $k<0$ the function $a(z)$ is a holomorphic function in the pointed open disc $\mathbf{D}(\rho(a)) \backslash\{0\}$. In particular, the function $a\left(t^{-1}\right)=t^{-k} b\left(t^{-1}\right)$ is a smooth function on the real open interval $(\sigma(a), \infty):=\{t \in \mathbb{R} ; t>\sigma(a)\}$, hence

$$
a\left(t^{-1}\right) \in \mathrm{C}^{\infty}(\sigma(a), \infty):=\{f:(\sigma(a), \infty) \rightarrow \mathbb{C} ; f \text { smooth }\}
$$

The sequences $a\left(t^{-1}\right), t \in \mathbb{N}, t>\sigma(a)$, are the time-varying coefficients of the difference equations of the present paper that in [4] were used for $a \in \mathbb{C}(z)=\mathbb{C}\left(z^{-1}\right)$. The coefficient functions $a\left(t^{-1}\right), a \in \mathbf{K}$, are of at most polynomial growth on each closed interval $\left[\sigma_{1}, \infty\right), \sigma_{1}>\sigma(a)$, i.e., there are $c>0$ and $m \in \mathbb{N}$ such that $\left|a\left(t^{-1}\right)\right| \leq c t^{m}$ for $t \geq \sigma_{1}[5,(29)]$. For nonzero $a$ there is $\sigma_{2}>\sigma(a)$ such that $a\left(t^{-1}\right) \neq 0$ for $t \geq \sigma_{2}$. These properties of the coefficient functions are essential for the module-behavior duality and for the definition and properties of exponential stability of autonomous behaviors.

Let, more generally, $A=\left(A_{\mu \nu}\right)_{1 \leq \mu, \nu \leq n} \in \mathbf{K}^{n \times n}$ be any square matrix and define

$$
\sigma(A):=\max \left\{\sigma\left(A_{\mu \nu}\right) ; 1 \leq \mu, v \leq n\right\}, \quad \rho(A):=\sigma(A)^{-1} .
$$

Then the function $t \mapsto A\left(t^{-1}\right)$ is a smooth matrix function on the open real interval $(\sigma(A), \infty)$. For $t_{0} \in \mathbb{N}$ we consider the signal space

$$
W\left(t_{0}\right):=\mathbb{C}^{t_{0}+\mathbb{N}}:=\left\{(w(t))_{t \geq t_{0}} ; t \in \mathbb{N}, w(t) \in \mathbb{C}\right\}
$$

of complex sequences or discrete signals starting at the initial time $t_{0}$. For $n \in \mathbb{N}$ we use the column spaces $\mathbb{C}^{n}$ and $W\left(t_{0}\right)^{n}$ and identify

$$
W\left(t_{0}\right)^{n}=\left(\mathbb{C}^{n}\right)^{t_{0}+\mathbb{N}} \ni\left(w_{1}, \ldots, w_{n}\right)^{\top}=\left(w\left(t_{0}\right), w\left(t_{0}+1\right), \ldots\right), w_{i}(t)=w(t)_{i}
$$

If $t_{0}>\sigma(A), t_{0} \in \mathbb{N}$, the matrix $A$ gives rise to the state space equation resp. the behavior or solution space

$$
\begin{aligned}
& x(t+1)=A\left(t^{-1}\right) x(t), t \in \mathbb{N}, t \geq t_{0}, \text { resp. } \\
& \mathcal{K}\left(A, t_{0}\right):=\left\{x \in W\left(t_{0}\right)^{n} ; \forall t \geq t_{0}: x(t+1)=A\left(t^{-1}\right) x(t)\right\} .
\end{aligned}
$$

The transition matrix $[15$, p. 392] associated to (9) is

$$
\Phi_{A}\left(t, t_{0}\right):=A\left((t-1)^{-1}\right) * \cdots * A\left(t_{0}^{-1}\right), t \geq t_{0}>\sigma(A), \Phi_{A}\left(t_{0}, t_{0}\right)=\mathrm{id}_{n} .
$$

There is the obvious isomorphism

$$
\mathcal{K}\left(A, t_{0}\right) \cong \mathbb{C}^{n}, x \mapsto x\left(t_{0}\right), x(t)=\Phi_{A}\left(t, t_{0}\right) x\left(t_{0}\right)
$$


For $\xi=\left(\xi_{1}, \ldots, \xi_{n}\right)^{\top} \in \mathbb{C}^{n}$ and $M \in \mathbb{C}^{n \times n}$ we use the maximum norms

$$
\|\xi\|:=\max _{i}\left|\xi_{i}\right| \text { and }\|M\|:=\max \left\{\|M \xi\| ; \xi \in \mathbb{C}^{n},\|\xi\|=1\right\}
$$

The system, i.e., the matrix $A$ and the equation and behavior from (9), are called exponentially stable (e.s.) [4, Def. 1.7, Cor. 3.3] if

$$
\begin{gathered}
\exists t_{0}>\sigma(A) \exists \alpha>0 \exists \text { p.g. } \varphi \in \mathbb{C}^{t_{0}+\mathbb{N}}, \varphi>0, \\
\forall t \geq t_{1} \geq t_{0}:\left\|\Phi_{A}\left(t, t_{1}\right)\right\| \leq \varphi\left(t_{1}\right) e^{-\alpha\left(t-t_{1}\right)} .
\end{gathered}
$$

Here a sequence $\varphi \in \mathbb{C}^{t_{0}+\mathbb{N}}$ is called of at most polynomial growth (p.g.) if $|\varphi(t)| \leq c t^{m}, t \geq t_{0}$, for some $c>0$ and $m \in \mathbb{N}$. In [4] we used the notation $\rho:=e^{-\alpha}<1$ and $\rho^{t-t_{1}}=e^{-\alpha\left(t-t_{1}\right)}$. In particular, e.s. implies asymptotic stability, i.e., $\lim _{t \rightarrow \infty} \Phi_{A}\left(t, t_{1}\right)=0$ for $t_{1} \geq t_{0}$. The system is called uniformly e.s. (u.e.s.) [15, Def. 22.5] if $\varphi$ in (13) can be chosen constant. Notice that (13) is a property of the behavior family $\left(\mathcal{K}\left(A, t_{1}\right)\right)_{t_{1} \geq t_{0}}$ and of the trajectories $x(t)=\Phi_{A}\left(t, t_{1}\right) x\left(t_{1}\right), t \geq t_{1} \geq t_{0}$, for sufficiently large $t_{0}$. This is appropriate for stability questions where the behavior of $x(t)$ for $t \rightarrow \infty$ is investigated. In [15] the author considers LTV state space equations $x(t+1)=F(t) x(t), t \geq 0$, with an arbitrary sequence of complex matrices $F=(F(0), F(1), \ldots) \in\left(\mathbb{C}^{n \times n}\right)^{\mathbb{N}}=\left(\mathbb{C}^{\mathbb{N}}\right)^{n \times n}$. All stability results in $[15$, Chs. 22-24] require additional properties of $F$. Our choice in [4] and in the present paper is

$$
F(t):=A\left(t^{-1}\right), A \in \mathbb{C}<<z>>^{n \times n} \supset \mathbb{C}(z)^{n \times n}, t>\sigma(A)
$$

A nonzero $A$ admits a unique representation

$$
A(z)=z^{-k} B(z), k \in \mathbb{Z}, B(z)=\sum_{i=0}^{\infty} B_{i} z^{i} \in \mathbb{C}<z>^{n \times n}, B_{i} \in \mathbb{C}^{n \times n}, B_{0} \neq 0,
$$

where the exponent $-k$ is chosen for notational convenience in Theorem 1.1.

Theorem 1.1 Consider a matrix $A(z) \in \mathbb{C}<<z>^{n \times n}$ and the state space system defined by the data from (9) to (10).

(i) If $A=\sum_{i=0}^{\infty} A_{i} z^{i}$ is a power series then the system is e.s. if and only if all eigenvalues of $A_{0}$ have absolute value $<1$.

(ii) If $k>0$ and $B_{0}$ is not nilpotent in (15) then the system is not e.s. and indeed

$$
\exists t_{0}>\sigma(A) \forall t_{1} \geq t_{0}: \sup _{t \geq t_{1}}\left\|\Phi_{A}\left(t, t_{1}\right)\right\|=\infty
$$


i.e., the system is unstable.

(iii) Assume $k>0$ and $B_{0}$ nilpotent in (15) and

$$
\begin{aligned}
\operatorname{det}(B(z)) & =b_{\ell} z^{\ell} c(z), \quad \ell \geq 1,0 \neq b_{\ell} \in \mathbb{C}, \\
c(z) & =1+c_{1} z+c_{2} z^{2}+\cdots \in \mathbb{C}<z>.
\end{aligned}
$$

If $k n>\ell$ then (16) holds and the system is not e.s.

The significance of Theorem 1.1 for arbitrary autonomous behaviors instead of state space behaviors follows from Corollary 3.11.

Example 1.2 That $B_{0}$ in item (ii) of Theorem 1.1 is not nilpotent cannot be omitted. To see this consider the nilpotent matrix $B_{0}:=\left(\begin{array}{ll}0 & 1 \\ 0 & 0\end{array}\right) \in \mathbb{C}^{2 \times 2}$. For $0 \neq \lambda \in \mathbb{C}$ and $\rho:=|\lambda|=e^{-\alpha}>0, \alpha \in \mathbb{R}$, define

$$
\begin{aligned}
A(z) & :=z^{-1} B(z), B(z):=B_{0}+z \lambda \mathrm{id}_{2} \Longrightarrow A\left(t^{-1}\right)=B_{0} t+\lambda \operatorname{id}_{2}=\left(\begin{array}{ll}
\lambda & t \\
0 & \lambda
\end{array}\right) \\
& \Longrightarrow \Phi_{A}\left(t, t_{0}\right)=\left(\begin{array}{cc}
\lambda^{t-t_{0}} & \lambda^{t-t_{0}-1} \sum_{i=t_{0}}^{t-1} i \\
0 & \lambda^{t-t_{0}}
\end{array}\right), \operatorname{det}(B(z))=\lambda^{2} z^{2}, \operatorname{det}\left(A\left(t^{-1}\right)\right)=\lambda^{2} .
\end{aligned}
$$

For $\rho \geq 1$ the sequence $\Phi_{A}\left(t, t_{0}\right)$ does not converge to zero and therefore the system is not e.s. The sum $\sum_{i=t_{0}}^{t-1} i$ grows polynomially. If $\rho=e^{-\alpha}<1$ or $\alpha>0$ the transition matrix $\Phi_{A}\left(t, t_{0}\right)$ decreases exponentially with a decay factor $e^{-\alpha^{\prime}\left(t-t_{0}\right)}$ for every $\alpha^{\prime}$ with $0<\alpha^{\prime}<\alpha$. So the system defined by $A$ is e.s. for $|\lambda|=e^{-\alpha}<1$.

Remark 1.3 The ring $\mathbb{C}<z>$ is defined by analytic conditions on the coefficients of the power series that imply its good algebraic properties. These are inherited by $\mathbf{K}$. Also the e.s. of an autonomous behavior is defined by analytic conditions on its trajectories [4, Def. 1.7]. In contrast, the construction of the category of behaviors and the derivation of the module-behavior duality proceed algebraically. This explains the necessity for both analytic and algebraic arguments in [4] and the present paper.

Notations and abbreviations $\mathbf{D}(\rho):=\{z \in \mathbb{C} ;|z|<\rho\}, \rho>0$, e.s. = exponentially stable, exponential stability, f.g. $=$ finitely generated, LTV $=$ linear time-varying, resp. $=$ respectively, u.e.s. $=$ uniformly e.s., w.e.s. $=$ weakly e.s., $X^{p \times q}=$ set of $p \times q$-matrices with entries in $X, X^{1 \times q}=$ rows, $X^{q}:=X^{q \times 1}=$ columns, $X^{\bullet \times \bullet}:=\bigcup_{p, q \geq 0} X^{p \times q}$.

\section{The proof of Theorem 1.1}

(i) Since $A$ is a power series we can write

$$
\begin{aligned}
A(z) & =A_{0}+z C(z) \in \mathbb{C}<z>^{n \times n}, \\
C(z) & =A_{1}+A_{2} z+\cdots \in \mathbb{C}<z>^{n \times n} \\
& \Longrightarrow A\left(t^{-1}\right)=A_{0}+t^{-1} C\left(t^{-1}\right), t \geq t_{0}>\sigma(A) .
\end{aligned}
$$

The function $C\left(t^{-1}\right)$ is bounded for $t \geq t_{0}$ and therefore $t^{-1} C\left(t^{-1}\right)$ is a disturbance term that is small for large $t$. 
(a) If $|\lambda|<1$ for all eigenvalues of $A_{0}$ the system $x(t+1)=A_{0} x(t), t \geq t_{0}$, is uniformly exponentially stable (u.e.s.). According to [15, Thm. 24.7], [4, Cor. 3.17] the equation $x(t+1)=A\left(t^{-1}\right) x(t), t \geq t_{0}$, is also u.e.s. and therefore e.s.

(b) Assume that $A_{0}$ has an eigenvalue $\lambda$ with $|\lambda|>1$. According to [4, Thm. 3.21] the system is exponentially unstable and, in particular,

$$
\begin{aligned}
\exists t_{0} & >\sigma(A) \exists \rho>1 \forall t \geq t_{1} \geq t_{0}:\left\|\Phi_{A}\left(t, t_{1}\right)\right\| \\
& \geq \rho^{t-t_{1}} \Longrightarrow \sup _{t \geq t_{1}}\left\|\Phi_{A}\left(t, t_{1}\right)\right\|=\infty .
\end{aligned}
$$

This implies that the system $x(t+1)=A\left(t^{-1}\right) x(t)$ is not e.s.

(c) Assume that $A_{0}$ has an eigenvalue $\lambda$ with $|\lambda|=1$ and that the system $x(t+$ $1)=A\left(t^{-1}\right) x(t), t>\sigma(A)$, is e.s. By (13)

$$
\begin{aligned}
& \exists t_{0}>\sigma(A) \exists \alpha>0, \rho:=e^{-\alpha}<1, \exists \text { p.g. } \varphi \in \mathbb{C}^{t_{0}+\mathbb{N}}, \varphi>0, \\
& \forall t \geq t_{1} \geq t_{0}:\left\|\Phi_{A}\left(t, t_{1}\right)\right\| \leq \varphi\left(t_{1}\right) \rho^{t-t_{1}} .
\end{aligned}
$$

Now consider the modified system

$$
\begin{aligned}
y(t+1) & =e^{\alpha} A\left(t^{-1}\right) y(t)=\rho^{-1} A\left(t^{-1}\right) y(t), t>\sigma(A), \text { with } \\
\rho^{-1} A(z) & =\left(\rho^{-1} A_{0}\right)+z\left(\rho^{-1} C(z)\right) .
\end{aligned}
$$

The matrix $\rho^{-1} A_{0}$ has the eigenvalue $\rho^{-1} \lambda$ with $\left|\rho^{-1} \lambda\right|=\rho^{-1}=e^{\alpha}>1$. From (b) we infer

$$
\begin{aligned}
\exists t_{2} & \geq t_{1} \forall t \geq t_{3} \geq t_{2}: \sup _{t \geq t_{3}}\left\|\Phi_{\rho^{-1} A}\left(t, t_{3}\right)\right\|=\infty . \text { But } \\
\Phi_{\rho^{-1} A}\left(t, t_{3}\right) & =\rho^{-\left(t-t_{3}\right)} \Phi_{A}\left(t, t_{3}\right) \\
& \Longrightarrow\left\|\Phi_{\rho^{-1} A}\left(t, t_{3}\right)\right\|=\rho^{-\left(t-t_{3}\right)}\left\|\Phi_{A}\left(t, t_{3}\right)\right\| \\
& \leq \rho^{-\left(t-t_{3}\right)}\left(\varphi\left(t_{3}\right) \rho^{t-t_{3}}\right)=\varphi\left(t_{3}\right) .
\end{aligned}
$$

The first and the last line of (23) are in contradiction and therefore $x(t+1)=$ $A\left(t^{-1}\right) x(t)$ cannot be e.s.

This completes the proof of part (i) of the theorem.

(ii) Recall that a square complex matrix is nilpotent if and only if 0 is its only eigenvalue. Under the conditions of (ii) the matrix $B_{0}$ has a nonzero eigenvalue $\lambda$. Choose $\rho>|\lambda|^{-1}$ so that $|\rho \lambda|>1$ for the eigenvalue $\rho \lambda$ of the matrix $\rho B_{0}$. 
According to (i)(b) $\rho B$ is exponentially unstable and indeed

$$
\begin{aligned}
\exists t_{0}>\sigma(A) & =\sigma(\rho B) \forall t \geq t_{1} \geq t_{0}: \sup _{t \geq t_{1}}\left\|\Phi_{\rho B}\left(t, t_{1}\right)\right\|=\infty . \text { But } \\
A\left(t^{-1}\right) & =t^{k} \rho^{-1}(\rho B)\left(t^{-1}\right), \Gamma\left(t, t_{1}\right):=(t-1) * \cdots * t_{1} \\
& \Longrightarrow \forall t \geq t_{1} \geq t_{0}: \Phi_{A}\left(t, t_{1}\right)=\Gamma\left(t, t_{1}\right)^{k} \rho^{-\left(t-t_{1}\right)} \Phi_{\rho B}\left(t, t_{1}\right) \\
& \Longrightarrow\left\|\Phi_{A}\left(t, t_{1}\right)\right\|=\Gamma\left(t, t_{1}\right)^{k} \rho^{-\left(t-t_{1}\right)}\left\|\Phi_{\rho B}\left(t, t_{1}\right)\right\| . \text { Further } \\
\Gamma\left(t, t_{1}\right)^{k} \rho^{-\left(t-t_{1}\right)} & =\frac{(t-1)^{k}}{\rho} * \cdots * \frac{t_{1}^{k}}{\rho} \underset{t \rightarrow \infty}{\longrightarrow} \infty \\
& \Longrightarrow \forall t_{1} \geq t_{0}: \sup _{t \geq t_{1}}\left\|\Phi_{A}\left(t, t_{1}\right)\right\|=\infty .
\end{aligned}
$$

(iii) Under the condition of Theorem 1.1, (iii), choose $t_{0}>\sigma(A)$ such that $\left|c\left(t^{-1}\right)\right| \geq$ $1 / 2$ for $t \geq t_{0}$. The determinant of

$$
\begin{aligned}
A(z) & =z^{-k} B(z) \text { is } \operatorname{det}(A(z))=z^{-k n} \operatorname{det}(B(z))=b_{\ell} z^{-(k n-\ell)} c(z) \\
& \Longrightarrow \forall t \geq t_{0}:\left|\operatorname{det}\left(A\left(t^{-1}\right)\right)\right|=\left|b_{\ell}\right| t^{k n-\ell}\left|c\left(t^{-1}\right)\right| \geq\left(\left|b_{\ell}\right| / 2\right) t^{k n-\ell} \\
& \Longrightarrow \forall t \geq t_{1} \geq t_{0}:\left|\operatorname{det}\left(\Phi_{A}\left(t, t_{1}\right)\right)\right| \geq \Gamma\left(t, t_{1}\right)^{k n-\ell}\left(\left|b_{\ell}\right| / 2\right)^{t-t_{1}} \underset{t \rightarrow \infty}{\longrightarrow} \infty
\end{aligned}
$$

where the last implication follows as in (24) due to $k n-\ell>0$. If the sequence $\left\|\Phi_{A}\left(t, t_{1}\right)\right\|, t \geq t_{1}$, was bounded so would be the sequence of determinants $\left|\operatorname{det}\left(\Phi_{A}\left(t, t_{1}\right)\right)\right|$.

\section{Laurent coefficients}

We explain the basic notions of a variant of the theory from [4] since we use the difference field $\mathbf{K}=\mathbb{C}<<z>>$ instead of the field $\mathbb{C}(z) \subset \mathbf{K}$ of rational functions in [4]. Recall $W\left(t_{0}\right)=\mathbb{C}^{t_{0}+\mathbb{N}}$ for $t_{0} \in \mathbb{N}$ from (7). The space $\mathbb{C}^{t_{0}+\mathbb{N}}=W\left(t_{0}\right)$ is also a difference $\mathbb{C}$-algebra with the componentwise multiplication and the shift algebra homomorphism

$$
\Phi_{d}: \mathbb{C}^{t_{0}+\mathbb{N}} \rightarrow \mathbb{C}^{t_{0}+\mathbb{N}}, c \mapsto \Phi_{d}(c), \Phi_{d}(c)(t)=c(t+1), t \geq t_{0}
$$

It gives rise to the noncommutative skew-polynomial algebra of difference operators [9, Section 1.2.3], [4, (20)]

$$
\mathbf{B}\left(t_{0}\right):=\mathbb{C}^{t_{0}+\mathbb{N}}\left[s ; \Phi_{d}\right]:=\bigoplus_{j=0}^{\infty} \mathbb{C}^{t_{0}+\mathbb{N}} s^{j}, s c=\Phi_{d}(c) s, c \in \mathbb{C}^{t_{0}+\mathbb{N}}
$$


The space $W\left(t_{0}\right)$ is a left $\mathbf{B}\left(t_{0}\right)$-module with the action $f \circ w$ for $f=\sum_{j=0}^{\infty} f_{j} s^{j} \in$ $\mathbf{B}\left(t_{0}\right)$ and $w \in W\left(t_{0}\right)[4,(21)]$, defined by

$$
(f \circ w)(t):=\sum_{j} f_{j}(t) w(t+j), t \geq t_{0}
$$

Of course, almost all, i.e., up to finitely many, $f_{j}$ are zero so that the sums $\sum_{j}$ are actually finite, here and in later occurrences. As usual the action is extended to the action $R \circ w[4,(22)]$ of a matrix

$$
\begin{aligned}
R & =\sum_{j} R_{j} s^{j} \in \mathbf{B}\left(t_{0}\right)^{p \times q}, R_{j} \in\left(\mathbb{C}^{t_{0}+\mathbb{N}}\right)^{p \times q}, \text { on } w \in W\left(t_{0}\right)^{q} \text { by } \\
(R \circ w)(t) & :=\sum_{j} R_{j}(t) w(t+j), t \geq t_{0} .
\end{aligned}
$$

The behavior or solution space defined by $R$ is

$$
\mathcal{B}\left(R, t_{0}\right):=\left\{w \in W\left(t_{0}\right)^{q} ; R \circ w=0\right\} .
$$

For $\sigma>0$ the algebra $\mathrm{C}^{\infty}(\sigma, \infty)$ is also a difference algebra with the algebra endomorphism

$$
\Phi_{s}: \mathrm{C}^{\infty}(\sigma, \infty) \rightarrow \mathrm{C}^{\infty}(\sigma, \infty), \Phi_{s}(f)(t):=f(t+1) .
$$

It gives rise to the skew-polynomial algebra

$$
\mathbf{A}_{s}(\sigma):=\mathrm{C}^{\infty}(\sigma, \infty)\left[s ; \Phi_{s}\right]:=\oplus_{j \in \mathbb{N}} \mathrm{C}^{\infty}(\sigma, \infty) s^{j}, s f=\Phi_{s}(f) s, f \in \mathrm{C}^{\infty}(\sigma, \infty) .
$$

For $t_{0}>\sigma$ the map

$$
\Psi_{s}: \mathrm{C}^{\infty}(\sigma, \infty) \rightarrow \mathbb{C}^{t_{0}+\mathbb{N}}, f \mapsto(f(t))_{t \geq t_{0}},
$$

is a difference algebra homomorphism since $\Phi_{d} \Psi_{s}=\Psi_{s} \Phi_{s}$ and therefore its extension

$$
\begin{aligned}
\Psi_{s}: \mathbf{A}_{s}(\sigma) & =\mathrm{C}^{\infty}(\sigma, \infty)\left[s ; \Phi_{s}\right] \rightarrow \mathbf{B}\left(t_{0}\right)=\mathbb{C}^{t_{0}+\mathbb{N}}\left[s ; \Phi_{d}\right], \\
\Psi_{s}\left(\sum_{j} f_{j} s^{j}\right) & :=\sum_{j} \Psi_{s}\left(f_{j}\right) s^{j},
\end{aligned}
$$

(denoted with the same letter) is an algebra homomorphism. The algebras $\mathbf{C}^{\infty}(\sigma, \infty)$ and $\mathbb{C}^{t_{0}+\mathbb{N}}$ are not noetherian and have many zero-divisors and therefore very little is known about the rings of difference operators from (27) to (32) and their modules. 
Therefore we restrict the time-varying coefficients of discrete difference equations to sequences

$$
a\left(t^{-1}\right)=t^{-k} \sum_{i=0}^{\infty} b_{i} t^{-i}, a=z^{k} b, b=\sum_{i=0}^{\infty} b_{i} z^{i} \in \mathbb{C}<z>
$$

where $t$ is chosen sufficiently large as explained in Lemma 3.1, for instance $t>$ $\sigma(a)=\rho(a)^{-1}$. In the latter case we have $(t+1)^{-1}=\frac{t^{-1}}{1+t^{-1}}<\rho(a)$ and

$$
\begin{aligned}
\forall t & >\sigma(a): a\left((t+1)^{-1}\right)=a\left(t^{-1}\left(t^{-1}+1\right)^{-1}\right) \\
& =(t+1)^{-k} \sum_{i=0}^{\infty} b_{i}(1+t)^{-i}=\left(\frac{t^{-1}}{t^{-1}+1}\right)^{k} \sum_{i=0}^{\infty} b_{i}\left(\frac{t^{-1}}{t^{-1}+1}\right)^{i} .
\end{aligned}
$$

This suggests to make $\mathbb{C}<<z>>$ a difference field [16, Ex. 1.2], [4, §4.7] via the field automorphism

$$
\Phi: \mathbb{C}<<z>>\stackrel{\cong}{\longrightarrow} \mathbb{C}<<z>>, \Phi(z)=z(1+z)^{-1}, \Phi\left(z^{-1}\right)=z^{-1}+1 .
$$

If

$$
\begin{aligned}
a & =z^{k} b, b=\sum_{i=0}^{\infty} b_{i} z^{i} \in \mathbb{C}<z>\text { then }(1+z)^{-i} \\
& =\sum_{j=0}^{\infty}\left(\begin{array}{c}
-i \\
j
\end{array}\right) z^{j} \in \mathbb{C}<z> \\
\text { and } \Phi(a) & :=a\left(\frac{z}{1+z}\right)=\left(\frac{z}{1+z}\right)^{k} \sum_{i=0}^{\infty} b_{i}\left(\frac{z}{1+z}\right)^{i} \\
& =z^{k}(1+z)^{-k} \sum_{i=0}^{\infty} b_{i} z^{i}(1+z)^{-i}
\end{aligned}
$$

The corresponding skew-polynomial algebra of difference operators is

$$
\mathbf{A}:=\mathbf{K}[s ; \Phi]=\oplus_{j \in \mathbb{N}} \mathbf{K} s^{j}, s a=\Phi(a) s .
$$

The $\mathbb{C}$-algebra $\mathbf{A}$ is a noncommutative euclidean domain $[9, \S 1.2]$, especially principal, and the f.g. left A-modules are precisely known [9, Thm. 1.2.9, § 5.7., Cor. 5.7.19]. The operators in $\mathbf{A}$ have the form $f:=\sum_{j} f_{j} s^{j} \in \mathbf{A}, f_{j} \in \mathbf{K}$, where almost all $f_{j}$ are zero. We define $\rho(f):=\min _{j}\left\{\rho\left(f_{j}\right) ; f_{j} \neq 0\right\}, \sigma(f):=\rho(f)^{-1}$.

In the sequel we make use of the equation $\Phi(a)\left(t^{-1}\right)=a\left((t+1)^{-1}\right)$ for $t>\sigma(a)$. Since $\rho(\Phi(a))$ may be smaller than $\rho(a)$, cf. Example 3.3, the left side of this equation 
is not defined a priori. To solve this problem we introduce difference subalgebras $\mathbf{K}(\rho) \subset \mathbf{K}, \rho>0$, such that the map $\Psi_{\rho}: \mathbf{K}(\rho) \rightarrow C^{\infty}\left(\rho^{-1}, \infty\right), a \mapsto a\left(t^{-1}\right)$, is a well-defined difference algebra homomorphism. We need the following detour: For an open set $U \subseteq \mathbb{C}$ let $\mathcal{O}(U)$ denote the $\mathbb{C}$-algebra of holomorphic functions in $U$. So any $a \in \mathbb{C}<<z>>$ defines the holomorphic function $a(z) \in \mathcal{O}(\mathbf{D}(\rho(a)) \backslash\{0\})$. In general, this can be extended to larger connected open sets. For $\rho>0$ we define the subset $\mathbf{K}(\rho) \subset \mathbf{K}$ as follows: An element $a \in \mathbf{K}$ belongs to $\mathbf{K}(\rho)$ if there is an open connected neighborhood $U(a)$ of 0 and a holomorphic function $f \in \mathcal{O}(U(a) \backslash\{0\})$ such that $[0, \rho) \subset U(a)$ and $f(z)=a(z)$ for $z \neq 0$ near 0 . In other terms, the germ of $f$ at 0 is $a$. Since $U(a) \backslash\{0\}$ is connected the function $f$ is unique with these properties, due to the identity theorem. The $\mathbf{K}(\rho)$ obviously satisfy

$$
a \in \mathbf{K}(\rho(a)), \text { hence } \mathbf{K}=\bigcup_{\rho>0} \mathbf{K}(\rho) \text { and } \forall \rho_{1} \leq \rho_{2}: \mathbf{K}\left(\rho_{2}\right) \subseteq \mathbf{K}\left(\rho_{1}\right) \text {. }
$$

All entire functions $a \in \mathcal{O}(\mathbb{C}) \subset \mathbb{C}<<z>>$ and $z^{-1}$ belong to all $\mathbf{K}(\rho)$.

Definition and Lemma 3.1 1. The value $a\left(t^{-1}\right):=f\left(t^{-1}\right)$ for $t>\rho^{-1}$ is independent of the choice of the extension $f \in \mathcal{O}(U(a) \backslash\{0\})$ of a.

2. The set $\mathbf{K}(\rho), \rho>0$, is a subalgebra of $\mathbf{K}$, i.e., additively and multiplicatively closed, and the map

$$
\Psi_{\rho}: \mathbf{K}(\rho) \rightarrow \mathrm{C}^{\infty}\left(\rho^{-1}, \infty\right), a \mapsto a\left(t^{-1}\right),
$$

is an algebra monomorphism

Proof 1. Let $f_{i} \in \mathcal{O}\left(U_{i} \backslash\{0\}\right), i=1,2$, be two such extensions. The (open) connected component $U_{3}$ of $U_{1} \bigcap U_{2}$ containing 0 also contains $[0, \rho)$. Since $f_{1}$ and $f_{2}$ are holomorphic on $U_{3} \backslash\{0\}$ and extend $a$ and since $U_{3} \backslash\{0\}$ is connected we conclude $\left.f_{1}\right|_{U_{3} \backslash\{0\}}=\left.f_{2}\right|_{U_{3} \backslash\{0\}}$ and hence $f_{1}\left(t^{-1}\right)=f_{2}\left(t^{-1}\right)$ for $t>\rho^{-1}$.

2. (a) For $a_{1}, a_{2} \in \mathbf{K}(\rho)$ the intersection $U\left(a_{1}\right) \bigcap U\left(a_{2}\right)$ is an open neighborhood of 0 and contains $[0, \rho)$. Let $f_{i}$ denote the holomorphic extensions of the $a_{i}$ to $U\left(a_{i}\right) \backslash\{0\}$ and $U_{3}$ the (open) connected component of $U\left(a_{1}\right) \bigcap U\left(a_{2}\right)$ containing 0 and hence $[0, \rho)$. The function $f_{1}+/ * f_{2}$ is holomorphic on $\left(U\left(a_{1}\right) \bigcap U\left(a_{2}\right)\right) \backslash$ $\{0\}$ and hence on $U_{3} \backslash\{0\}$ and obviously coincides with $a_{1}+/ * a_{2}$ near zero, hence $a_{1}+/ * a_{2} \in \mathbf{K}(\rho)$.

For $t>\rho^{-1}$ this implies

$$
\begin{aligned}
\left(a_{1}+/ * a_{2}\right)\left(t^{-1}\right): & =\left(f_{1}+/ * f_{2}\right)\left(t^{-1}\right)=f_{1}\left(t^{-1}\right)+/ * f_{2}\left(t^{-1}\right) \\
& =a_{1}\left(t^{-1}\right)+/ * a_{2}\left(t^{-1}\right)
\end{aligned}
$$

Hence $\mathbf{K}(\rho)$ is a subalgebra of $\mathbf{K}$ and $\Psi_{\rho}$ is an algebra homomorphism.

(2)(b) $\Psi_{\rho}$ is injective: If $a\left(t^{-1}\right)=f\left(t^{-1}\right)=0$ for $t>\rho^{-1}$ the holomorphic function $f$ is zero on $(0, \rho)$. By means of the identity theorem this implies $f=0$ and $a=0$.

Lemma 3.2 For all $a \in \mathbf{K}$ and $m \in \mathbb{N}$ the following assertions hold: 
1. For all $a \in \mathbf{K}: \Phi^{m}(a)=a\left(z(1+m z)^{-1}\right)$.

2. For $\rho>0, m \in \mathbb{N}$ and $a \in \mathbf{K}(\rho)$ :

$$
\begin{aligned}
& \Phi^{m}(a) \in \mathbf{K}\left(\rho_{1}\right) \subset \mathbf{K}(\rho), \rho_{1}:=\left\{\begin{array}{ll}
\infty & \text { if } m \rho \geq 1 \\
\rho(1-m \rho)^{-1} & \text { if } m \rho<1
\end{array} \geq \rho,\right. \text { and } \\
& \forall t>\rho_{1}^{-1}=\left\{\begin{array}{ll}
0 & \text { if } \rho m \geq 1 \\
\rho^{-1}-m & \text { if } m \rho<1
\end{array}: \Phi^{m}(a)\left(t^{-1}\right)=a\left((t+m)^{-1}\right) .\right.
\end{aligned}
$$

With Lemma 3.1 this implies that $\mathbf{K}(\rho)$ is a difference subalgebra of $\mathbf{K}$, i.e. $\Phi(\mathbf{K}(\rho)) \subseteq \mathbf{K}(\rho)$. Moreover $\Psi_{\rho}$ is a morphism of difference algebras, i.e.,

$$
\Phi_{s} \Psi_{\rho}=\Psi_{\rho} \Phi \text { or } \forall t>\rho^{-1}: \Phi(a)\left(t^{-1}\right)=a\left((t+1)^{-1}\right) .
$$

Proof 1. The equation follows by induction from

$$
\begin{aligned}
\Phi^{m+1}(a) & =\Phi^{m}(a)\left(z(1+z)^{-1}\right) \\
& =a\left(\frac{z}{1+z} \frac{1}{1+m z(1+z)^{-1}}\right)=a\left(\frac{z}{1+(m+1) z}\right) .
\end{aligned}
$$

2. Let $f \in \mathcal{O}(U(a) \backslash\{0\})$ be a holomorphic extension of $a$ with $U(a) \supset[0, \rho)$. Consider the projective line $\overline{\mathbb{C}}=\mathbb{C} \uplus\{\infty\}$. For $m \geq 0$ there are the inverse biholomorphic maps

$$
\overline{\mathbb{C}} \cong \overline{\mathbb{C}}, z=w(1-m w)^{-1} \leftrightarrow w=z(1+m z)^{-1} .
$$

They induce the inverse biholomorphic maps

$$
\begin{aligned}
\overline{\mathbb{C}} \backslash\{-1 / m, \infty\} & =\mathbb{C} \backslash\{-1 / m\} \cong \overline{\mathbb{C}} \backslash\{1 / m, \infty\}=\mathbb{C} \backslash\{1 / m\} \text { and } \\
V & :=\left\{z \in \mathbb{C} ; z(1+m z)^{-1} \in U(a)\right\} \cong U(a) \backslash\{1 / m\} \subset U(a)
\end{aligned}
$$

Since $U(a)$ and $U(a) \backslash\{1 / m\}$ are open and connected the set $V$ is a connected open neighborhood of 0 . The function $f\left(z(1+m z)^{-1}\right)$ is holomorphic on $V \backslash\{0\}$ and coincides with $\Phi^{m}(a)(z)=a\left(z(1+m z)^{-1}\right)$ for small $z \neq 0$. This means that it is a holomorphic extension of $\Phi^{m}(a)$ or that $\Phi^{m}(a)$ is its germ at 0 . For $z>0$ the following equivalences hold:

$$
z(1+m z)^{-1} \in(0, \rho) \Longleftrightarrow z(1-m \rho)<\rho \Longleftrightarrow z \in\left(0, \rho_{1}\right)
$$

Hence

$$
\begin{aligned}
& \forall z \in\left(0, \rho_{1}\right): m^{-1} \neq z(1+m z)^{-1} \in(0, \rho) \subset U(a) \\
& \underset{(44)}{\Longrightarrow} z \in V \Longrightarrow\left(0, \rho_{1}\right) \subset V \Longrightarrow \Phi^{m}(a) \in \mathbf{K}\left(\rho_{1}\right) \subseteq \mathbf{K}(\rho) .
\end{aligned}
$$


For $t>\rho_{1}^{-1}=\left\{\begin{array}{ll}0 & \text { if } \rho m \geq 1 \\ \rho^{-1}-m & \text { if } m \rho<1\end{array}\right.$ or $t^{-1}<\rho_{1}$ this implies

$$
\begin{aligned}
(t+m)^{-1} & =\frac{t^{-1}}{1+m t^{-1}}<\rho \text { and } \\
\Phi^{m}(a)\left(t^{-1}\right) & =f\left(\frac{t^{-1}}{1+m t^{-1}}\right)=a\left(\frac{t^{-1}}{1+m t^{-1}}\right)=a\left((t+m)^{-1}\right) .
\end{aligned}
$$

\section{Example 3.3 Let}

$$
m:=1,0<\alpha<\rho<1 \text { and } f(z):=((z+\alpha)(z-\rho))^{-1} \in \mathcal{O}(\mathbb{C} \backslash\{-\alpha, \rho\}) .
$$

For the germ $a \in \mathbb{C}<z>$ of $f$ at 0 we get

$$
\begin{aligned}
\rho(a) & =\alpha,(0, \rho) \subset U(a):=\mathbb{C} \backslash\{-\alpha, \rho\}, \forall t>\rho^{-1}: a\left(t^{-1}\right)=f\left(t^{-1}\right), \\
f\left(\frac{z}{1+z}\right) & =-\alpha^{-1} \rho^{-1}(1+z)^{2}\left(1+\frac{1+\alpha}{\alpha} z\right)^{-1}\left(1-\rho_{1}^{-1} z\right)^{-1} \\
& \in \mathcal{O}\left(\mathbb{C} \backslash\left\{-\frac{\alpha}{1+\alpha}, \rho_{1}\right\}\right) \\
\rho(\Phi(a)) & =\frac{\alpha}{1+\alpha}<\alpha<\rho<\rho_{1}:=\frac{\rho}{1-\rho},\left(0, \rho_{1}\right) \subset \mathbb{C} \backslash\left\{-\frac{\alpha}{1+\alpha}, \rho_{1}\right\} \\
\forall t>\rho_{1}^{-1} & =\rho^{-1}-1: t+1>\rho^{-1} \text { and } \\
\Phi(a)\left(t^{-1}\right) & =f\left(\frac{t^{-1}}{1+t^{-1}}\right)=f\left((t+1)^{-1}\right)=a\left((t+1)^{-1}\right) .
\end{aligned}
$$

Remark 3.4 The preceding example can be generalized. Assume that $a \in \mathbb{C}<<$ $z>>$ can be extended to $f \in \mathcal{O}(\mathbb{C} \backslash S)$ where $S$ is a discrete closed subset of $\mathbb{C}$ and $S$ is the set of singularities of $f$. So $a$ is the germ of $f$ at zero, $U:=\mathbb{C} \backslash S$ is connected and both $S$ and $f$ are uniquely determined by $a$. Therefore $a(z):=f(z), z \in U$, is well-defined. In particular, $a\left(t^{-1}\right)$ is well-defined for $t \in \mathbb{N}$ and $t^{-1} \in(0, \rho) \cap U$. The inclusion $(0, \rho) \subset U$ is not required in this case, but was essential for the definition and properties of $\mathbf{K}(\rho)$.

The Lemmas 3.1 and 3.2 imply that

$$
\mathbf{A}(\rho):=\mathbf{K}(\rho)[s ; \Phi]=\bigoplus_{i \in \mathbb{N}} \mathbf{K}(\rho) s^{i} \subset \mathbf{A}=\mathbf{K}[s ; \Phi]
$$

is a subalgebra of $\mathbf{A}$ and that the extended map, denoted by the same letter,

$$
\begin{aligned}
\Psi_{\rho}: \mathbf{A}(\rho) & =\mathbf{K}(\rho)[s ; \Phi] \rightarrow \mathbf{A}_{s}\left(\rho^{-1}\right) \\
& =\mathbf{C}^{\infty}\left(\rho^{-1}, \infty\right)\left[s ; \Phi_{s}\right], a(z) s^{j} \mapsto a\left(t^{-1}\right) s^{j},
\end{aligned}
$$


is an algebra monomorphism. Since any $f=\sum_{j} f_{j} s^{j}$ belongs to $\mathbf{K}(\rho(f)), \rho(f)=$ $\min \left\{\rho\left(f_{j}\right) ; f_{j} \neq 0\right\}$, we again have $\mathbf{A}=\bigcup_{\rho>0} \mathbf{A}(\rho)$.

For $t_{0}>\rho^{-1}$ we compose $\Psi_{\rho}$ and $\Psi_{s}$ and obtain the algebra homomorphism

$$
\begin{aligned}
& \Psi:=\Psi_{s} \Psi_{\rho}: \mathbf{K}(\rho) \stackrel{\Psi_{\rho}}{\longrightarrow} \mathrm{C}^{\infty}\left(\rho^{-1}, \infty\right) \stackrel{\Psi_{s}}{\longrightarrow} \quad \mathbb{C}^{t_{0}+\mathbb{N}} \\
& \bigcap \underset{\Psi_{\rho}}{\bigcap} \quad \Psi_{\Psi_{s}} \quad \cap \\
& \Psi:=\Psi_{s} \Psi_{\rho}: \mathbf{A}(\rho) \stackrel{\Psi_{\rho}}{\longrightarrow} \quad \mathbf{A}_{s}\left(\rho^{-1}\right) \quad \stackrel{\Psi_{s}}{\longrightarrow} \quad \mathbf{B}\left(t_{0}\right) \\
& a(z) s^{j} \mapsto \quad a\left(t^{-1}\right) s^{j} \quad \mapsto\left(a\left(t^{-1}\right)\right)_{t \geq t_{0}} s^{j}
\end{aligned}
$$

Lemma $3.5 \Psi$ is injective.

Proof Since $\Psi$ is a homomorphism and $z$ is a unit in $\mathbf{K}(\rho)$ it suffices to show that for a power series $a \in \mathbf{K}(\rho)$ the equation $\Psi(a)=\left(a\left(t^{-1}\right)\right)_{t \geq t_{0}}=0$ implies $a=0$. This holds by the identity theorem since 0 is an accumulation point of the sequence $t^{-1}$.

Definition and Corollary 3.6 For $\rho>0$ and $t_{0}>\rho^{-1}$ the algebra monomorphism $\Psi$ and the left $\mathbf{B}\left(t_{0}\right)$ - module structure of $W\left(t_{0}\right)=\mathbb{C}^{t_{0}+\mathbb{N}}$ from (28) imply the action $\circ$ of $\mathbf{A}(\rho)$ on $W\left(t_{0}\right)$ defined by

$$
\begin{gathered}
f \circ w:=\Psi(f) \circ w, f=\sum_{j} f_{j} s^{j} \in \mathbf{A}(\rho), w \in W\left(t_{0}\right), \\
\forall t \geq t_{0}>\rho^{-1}:(f \circ w)(t)=\sum_{j} f_{j}\left(t^{-1}\right) w(t+j)
\end{gathered}
$$

This action makes $W\left(t_{0}\right)$ a left $\mathbf{A}(\rho)$-module, in particular $(f g) \circ w=f \circ(g \circ w)$ holds for $f, g \in \mathbf{A}(\rho)$ and $w \in \mathbb{C}^{t_{0}+\mathbb{N}}, t_{0}>\rho^{-1}$.

The homomorphisms $\Phi, \Phi_{d}, \Psi$ are extended to matrices componentwise. For a matrix $R=\sum_{j} R_{j} s^{j} \in \mathbf{A}^{p \times q}, R_{j} \in \mathbf{K}^{p \times q}$, we define, cf. (6),

$\rho(R):=\min \left\{\rho\left(R_{j}\right) ; R_{j} \neq 0\right\}, \sigma(R)=\rho(R)^{-1}$, and obtain $R \in \mathbf{A}(\rho(R))^{p \times q}$.

A matrix $R \in \mathbf{A}(\rho)^{p \times q}$ and $t_{0}>\rho^{-1}$ give rise to

$$
\Psi(R)=\sum_{j}\left(R_{j}\left(t^{-1}\right)\right)_{t \geq t_{0}} s^{j} \in \mathbf{B}\left(t_{0}\right)^{p \times q}=\bigoplus_{j}\left(\mathbb{C}^{t_{0}+\mathbb{N}}\right)^{p \times q} s^{j}
$$

and the solution space or behavior

$$
\begin{aligned}
\mathcal{B}\left(R, t_{0}\right): & =\left\{w \in W\left(t_{0}\right)^{q} ; R \circ w=0\right\}=\left\{w \in W\left(t_{0}\right)^{q} ; \Psi(R) \circ w=0\right\} \\
& =\left\{w \in W\left(t_{0}\right)^{q} ; \forall t \geq t_{0}: \sum_{j} R_{j}\left(t^{-1}\right) w(t+j)=0\right\} .
\end{aligned}
$$


As in Definition and Corollary 3.6 the equation

$$
(R S) \circ w=R \circ(S \circ w), R, S \in \mathbf{A}(\rho)^{p \times q}, w \in W\left(t_{0}\right)^{q}, t_{0}>\rho^{-1},
$$

holds and is decisive for the duality theory, recalled below.

For $A \in \mathbf{K}(\rho)^{q \times q}$ the state space behavior from (9) now obtains the form

$$
\mathcal{B}\left(s \operatorname{id}_{q}-A, t_{0}\right)=\mathcal{K}\left(A, t_{0}\right)=\left\{x \in W\left(t_{0}\right)^{q} ; \forall t \geq t_{0}: x(t+1)=A\left(t^{-1}\right) x(t)\right\} .
$$

Remark 3.7 Notice that $W\left(t_{0}\right)$ is not an $\mathbf{A}$-, but only an $\mathbf{A}(\rho)$-left module for $t_{0}>\rho^{-1}$. In contrast to the well-known algebraic structure of $\mathbf{A}$ that of $\mathbf{A}(\rho)$ and its f.g. left modules is unknown. To enable a module-behavior duality between f.g. A-left modules and behaviors those from (50) have to be modified as in [4, (7), (9)], see (54)-(65) below.

The subspace $\mathbb{C}^{(\mathbb{N})} \subset \mathbb{C}^{\mathbb{N}}$ consists of the signals $w \in \mathbb{C}^{\mathbb{N}}$ with finite support $\operatorname{supp}(w):=\{t \in \mathbb{N} ; w(t) \neq 0\}$. The factor space $W(\infty):=\mathbb{C}^{\mathbb{N}} / \mathbb{C}^{(\mathbb{N})}$ is an A-left module with the action $f \circ\left(w+\mathbb{C}^{(\mathbb{N})}\right):=v+\mathbb{C}^{(\mathbb{N})}$ where

$$
f=\sum_{j} f_{j} s^{j} \in \mathbf{A}, v(t):=\left\{\begin{array}{ll}
\sum_{j} f_{j}\left(t^{-1}\right) w(t+j) & \text { if } t>\sigma(f) \\
0 & \text { if } t \leq \sigma(f)
\end{array} .\right.
$$

This signal space $W(\infty)$ was already defined in [16, p. 5]. In [3, Thm. 839] it was shown for the coefficient field $\mathbb{C}(z)$ instead of $\mathbb{C}<<z>>$ here that it is a large injective Acogenerator and thus enables a module-behavior duality. This signal module $W(\infty)$ is unsuitable for the stability theory of LTV systems since the signals $w+\mathbb{C}^{(\mathbb{N})}$ do not have well-defined values $w(t) \in \mathbb{C}$ and in particular no initial value $w\left(t_{0}\right)$. So (uniform) exponential stability of state space behaviors as in (13) or of general behaviors [4, Def. 1.7] cannot be defined.

However, $W(\infty)$ is even a commutative ring since $\mathbb{C}^{(\mathbb{N})}$ is an ideal of $\mathbb{C}^{\mathbb{N}}$ with the componentwise multiplication. The shift on $\mathbb{C}^{\mathbb{N}}$ induces that on $W(\infty)$ and makes it a difference ring. The ring homomorphism

$$
\widetilde{\Psi}: \mathbf{K} \rightarrow W(\infty), a \mapsto \widetilde{\Psi}(a):=\widetilde{a}+\mathbb{C}^{(\mathbb{N})}, \widetilde{a}(t):= \begin{cases}a\left(t^{-1}\right) & \text { if } t>\sigma(a) \\ 0 & \text { if } t \leq \sigma(a)\end{cases}
$$

is well-defined, injective and indeed a monomorphism of difference rings. The algebra $W(\infty)$ and the preceding homomorphism can be used instead of $\Psi: \mathbf{K}(\rho) \rightarrow \mathbb{C}^{t_{0}+\mathbb{N}}$ from (47) to derive the duality theory, recalled below, with different, but similar arguments.

Two behavior families

$$
\mathcal{B}_{i}:=\left(\mathcal{B}\left(R_{i}, t_{0}\right)\right)_{t_{0}>\rho_{i}^{-1}}, \quad R_{i} \in \mathbf{A}\left(\rho_{i}\right)^{p_{i} \times q} \subset \mathbf{A}^{p_{i} \times q}, \quad i=1,2,
$$


are called equivalent, cf. [4, (7)], if

$$
\exists t_{1}>\max \left(\rho_{1}^{-1}, \rho_{2}^{-1}\right) \forall t_{2} \geq t_{1}: \mathcal{B}\left(R_{1}, t_{2}\right)=\mathcal{B}\left(R_{2}, t_{2}\right) .
$$

Since $t_{1}$ can be chosen large one may always assume that $\rho_{i}=\rho\left(R_{i}\right)$ for $i=1,2$. The equivalence class of $\mathcal{B}_{1}$ is denoted by $\operatorname{cl}\left(\mathcal{B}_{1}\right)$. The study of this class means to study the behaviors $\mathcal{B}\left(R_{1}, t_{2}\right)$ for large $t_{2}$. This is appropriate for stability questions where the trajectories $w(t)$ of a behavior are studied for $t \rightarrow \infty$.

If $M$ is a f.g. A-module with a given list $\mathbf{w}:=\left(\mathbf{w}_{1}, \ldots, \mathbf{w}_{q}\right)^{\top}$ of generators there is the canonical isomorphism

$$
\begin{aligned}
\mathbf{A}^{1 \times q} / U & \cong M, \xi+U \mapsto \xi \mathbf{w}=\sum_{i} \xi_{i} \mathbf{w}_{i} \text { where } \\
\xi & =\left(\xi_{1}, \ldots, \xi_{q}\right) \in \mathbf{A}^{1 \times q}, U:=\left\{\xi \in \mathbf{A}^{1 \times q} ; \xi \mathbf{w}=0\right\} .
\end{aligned}
$$

Since $\mathbf{A}$ is noetherian the submodule $U$ is f.g. and thus generated by the rows of some matrix $R \in \mathbf{A}^{p \times q}$, i.e., $U=\mathbf{A}^{1 \times p} R$. Since $\mathbf{A}$ is even a principal ideal domain $U$ is free and one may assume that $\operatorname{dim}_{\mathbf{A}}(U)=\operatorname{rank}(R)=p$. The matrix $R$ gives rise to behaviors

$$
\mathcal{B}\left(R, t_{0}\right), t_{0}>\sigma(R)=\rho(R)^{-1} \text {, and their class } \operatorname{cl}\left(\left(\mathcal{B}\left(R, t_{0}\right)\right)_{t_{0}>\sigma(R)}\right) .
$$

Equation (51) is used to show that this class depends on $U$ only and not on the special choice of $R$ [4, Lemma 2.5]. The class is denoted by

$$
\mathcal{B}(U):=\operatorname{cl}\left(\left(\mathcal{B}\left(R, t_{0}\right)\right)_{t_{0}>\sigma(R)}\right), U=\mathbf{A}^{1 \times p} R \subseteq \mathbf{A}^{1 \times q},
$$

and called the behavior defined by $U$. These behaviors $\mathcal{B}(U)$ were introduced in [4, (9)] for the coefficient field $\mathbb{C}(z)=\mathbb{C}\left(z^{-1}\right)$ of rational functions instead of $\mathbf{K} \supset \mathbb{C}(z)$. In particular, a matrix $A \in \mathbf{K}^{n \times n}$ gives rise to the solution spaces $\mathcal{B}\left(s \mathrm{id}_{n}-A, t_{0}\right)=$ $\mathcal{K}\left(A, t_{0}\right)$ from (9) to (52) and the Kalman state space behavior

$$
\mathcal{B}(U)=\operatorname{cl}\left(\left(\mathcal{K}\left(A, t_{0}\right)\right)_{t_{0}>\sigma(A)}\right), A \in \mathbf{K}^{n \times n}, U=\mathbf{A}^{1 \times n}\left(s \operatorname{id}_{n}-A\right) .
$$

Remark 3.8 The following three properties of the coefficient sequences $\left(a\left(t^{-1}\right)\right)_{t>\sigma(a)}, a \in \mathbf{K}$, are decisive:

1. Any nonzero $a \in \mathbb{C}<z>$ can be written as $a=a_{0}+z c(z)$ where $c(z)$ is bounded for $|z| \leq \rho<\rho(a)$ and hence $a\left(t^{-1}\right)=a_{0}+t^{-1} c\left(t^{-1}\right)$ with bounded $c\left(t^{-1}\right)$ for $t \geq t_{0}>\sigma(a)$. For large $t$ the term $t^{-1} c\left(t^{-1}\right)$ is a small disturbance of the constant $a_{0}$ and thus the perturbation results [15, Thm. 24.7], [4, Lemma 3.15] are applicable.

2. The sequences are of at most polynomial growth, indeed

$$
\begin{aligned}
a= & z^{-m} b, m \geq 0, b \in \mathbb{C}<z> \\
& \quad \text { implies } \exists c>0 \forall t \geq t_{0}>\sigma(a):\left|a\left(t^{-1}\right)\right| \leq c t^{m} .
\end{aligned}
$$


3. The sequences have no zeros for large $t$, i.e., $\exists t_{1}>\sigma(a) \forall t \geq t_{1}: a\left(t^{-1}\right) \neq 0$.

Result 3.9 (Meta-theorem) With the obvious necessary modifications all essential notions and results from [4] hold for the coefficient field $\mathbf{K}$ and the behaviors $\mathcal{B}(U)$ defined in (54).

For the preceding result one checks that the proofs of [4] use the properties of Remark 3.8 only. In particular, there is a canonical definition of behavior morphisms: If $M_{i}=\mathbf{A}^{1 \times q_{i}} / U_{i}, U_{i}=\mathbf{A}^{1 \times p_{i}} R_{i}, R_{i} \in \mathbf{A}^{p_{i} \times q_{i}}, i=1,2$, are two modules any $\mathbf{A}$ linear map $\varphi: M_{1} \rightarrow M_{2}$ has the form [4, (47), (48)]

$$
\begin{aligned}
& \varphi=(\circ P)_{\text {ind }}: M_{1} \rightarrow M_{2}, \xi+U_{1} \mapsto \xi P+U_{2}, \text { where } \\
& P \in \mathbf{A}^{q_{1} \times q_{2}}, \exists X \in \mathbf{A}^{p_{1} \times p_{2}} \text { with } R_{1} P=X R_{2} .
\end{aligned}
$$

If $R_{1}, R_{2}, P, X \in \mathbf{A}(\rho)^{\bullet \times \bullet}$, for instance if $\rho<\min \left(\rho\left(R_{1}\right), \rho\left(R_{2}\right), \rho(P), \rho(X)\right)$, and if $t_{0}>\rho^{-1}$ Eq. 51 implies

$$
\begin{aligned}
\forall w \in W\left(t_{0}\right)^{q_{2}}: R_{1} \circ(P \circ w)=X \circ\left(R_{2} \circ w\right) \\
\quad \Longrightarrow P \circ: \mathcal{B}\left(R_{2}, t_{0}\right) \rightarrow \mathcal{B}\left(R_{1}, t_{0}\right), w_{2} \mapsto P \circ w_{2}
\end{aligned}
$$

The equivalence class $\mathcal{B}(\varphi):=\operatorname{cl}\left(\left(P \circ: \mathcal{B}\left(R_{2}, t_{0}\right) \rightarrow \mathcal{B}\left(R_{1}, t_{0}\right)\right)_{t_{0}>\rho^{-1}}\right)$ is defined in analogy to (54), cf. [4, (33), (50)], and defines the behavior morphism

$$
\mathcal{B}(\varphi): \mathcal{B}\left(U_{2}\right) \rightarrow \mathcal{B}\left(U_{1}\right) .
$$

The map $\varphi$ is an isomorphism if and only if $\mathcal{B}(\varphi)$ is one, i.e., if $P \circ: \mathcal{B}\left(R_{2}, t_{1}\right) \rightarrow$ $\mathcal{B}\left(R_{1}, t_{1}\right)$ is an isomorphism for sufficiently large $t_{1}$.

Corollary 3.10 (cf. [4, Cor. 2.7, Thm. 1.6.]) The behaviors $\mathcal{B}(U)$ with these morphisms form an abelian category and the assignment $M=\mathbf{A}^{1 \times q} / U \mapsto \mathcal{B}(U)$ is a categorical duality or contravariant equivalence from $f . g$. A-modules $M$ with a given finite list of generators to behaviors.

A f.g. module $M=\mathbf{A}^{1 \times q_{1}} / U_{1}$ is a torsion module, cf. [4, $\left.\$ 2.5\right]$, if and only if $n:=\operatorname{dim}_{\mathbf{K}}(M)<\infty$ or if and only if it is isomorphic to a module in state space form, i.e.,

$$
\begin{aligned}
M & =\mathbf{A}^{1 \times q_{1}} / U_{1} \cong M_{2}=\mathbf{A}^{1 \times n} / U_{2}, U_{2}=\mathbf{A}^{1 \times n}\left(s \operatorname{id}_{n}-A\right), A \in \mathbf{K}^{n \times n} \\
\mathcal{B}\left(U_{2}\right) & =\operatorname{cl}\left(\left(\mathcal{K}\left(A, t_{0}\right)\right)_{t_{0}>\sigma(A)}\right) \cong \mathcal{B}\left(U_{1}\right) .
\end{aligned}
$$

The behavior

$$
\mathcal{B}(U)=\operatorname{cl}\left(\left(\mathcal{B}\left(R, t_{0}\right)\right)_{t_{0}>\sigma(R)}\right) \text { with } R \in \mathbf{A}^{p \times q}, U=\mathbf{A}^{1 \times p} R, M=\mathbf{A}^{1 \times q} / U
$$

is called autonomous if and only if there is $t_{1}>\sigma(R)$ such that all trajectories $w \in \mathcal{B}\left(R, t_{2}\right), t_{2} \geq t_{1}$, are determined by the initial vector $\left(w\left(t_{2}\right), \ldots, w\left(t_{2}+d\right)\right)$ 
of some fixed length $d$. This is the case if and only if the module $M=\mathbf{A}^{1 \times q} / U$ is a torsion module [4, Thm. 3.18].

Exponential stability (e.s.) of an autonomous behavior $\mathcal{B}(U)$ and of its torsion module $M=\mathbf{A}^{1 \times q} / U$ [4, Def. 1.7] is defined by a more general version of (13) and preserved by behavior isomorphisms $[4, \S 3.2]$. The state space behavior $\mathcal{B}\left(U_{2}\right)$ in (64) is e.s. if and only if the matrix $A$ is e.s. in the sense of (13) [4, Cor. 3.3].

Corollary 3.11 In the situation of (64) the general autonomous behavior $\mathcal{B}\left(U_{1}\right)$ is e.s. if and only if the matrix $A$ is e.s. in the sense of (13). The matrix $A$ and an explicit isomorphism $M_{1} \cong M_{2}$ or $\mathcal{B}\left(U_{2}\right) \cong \mathcal{B}\left(U_{1}\right)$ can be computed with the OreModules package [6,14]. Hence Theorem 1.1 describes an algorithmic test of e.s. for all autonomous behaviors except those for which the assumptions of Theorem 1.1, (ii), (iii), are not satisfied by $A$.

Corollary 3.12 ([4, Thms. 1.8, 3.11]) The e.s. behaviors and modules form Serre categories, i.e., are closed under subobjects, factor objects and extensions .

\section{Puiseux series and weak exponential stability}

To a large extent Theorem 1.1 can be extended to the difference field $\mathbf{P}$ of locally convergent Puiseux series, cf. [5, §3.1], where

$$
\mathbf{P}:=\bigcup_{m \geq 1} \mathbb{C}<<z^{1 / m}>>; \Phi(z)=z(1+z)^{-1}, \Phi\left(z^{1 / m}\right)=z^{1 / m}(1+z)^{-1 / m} .
$$

and its $\Phi$-invariant Bézout and valuation subdomain

$$
\mathbf{L}:=\bigcup_{m \geq 1} \mathbb{C}<z^{1 / m}>
$$

The field $\mathbf{P}$ is the algebraic closure of $\mathbf{K}=\mathbb{C}<<z>>$ [11]. If $m_{1}$ divides $m_{2}$ then $z^{1 / m_{1}}=\left(z^{1 / m_{2}}\right)^{m_{2} / m_{1}}$ and hence $\mathbb{C}<z^{1 / m_{1}}>\subseteq \mathbb{C}<z^{1 / m_{2}}>$. The nonzero elements of $\mathbb{C}<<z^{1 / m}>>$ have the unique form

$$
\begin{aligned}
a\left(z^{1 / m}\right) & =z^{k / m} b\left(z^{1 / m}\right), a=z^{k} b \in \mathbb{C}<<z>>, \\
k \in \mathbb{Z}, b & =\sum_{i} b_{i} z^{i} \in \mathbb{C}<z>, b_{0} \neq 0 .
\end{aligned}
$$

Such an $a\left(z^{1 / m}\right)$ induces the smooth function $a\left(t^{-1 / m}\right)=t^{-k / m} \sum_{i} b_{i} t^{-i / m}$ on the real interval $\left(\sigma(a)^{m}, \infty\right)$. The coefficient rings give rise to the operator domains

$$
\mathbf{L}[s ; \Phi] \subseteq \mathbf{B}:=\mathbf{P}[s ; \Phi] ;=\oplus_{j \in \mathbb{N}} \mathbf{P}^{j}
$$


Again $\mathbf{B}$ is a left and right euclidean domain. A matrix

$$
\begin{aligned}
R & =\sum_{j} R_{j}\left(z^{1 / m}\right) s^{j} \in \mathbf{B}^{p \times q} \text { with } R_{j} \\
& =\left(R_{j \mu \nu}(z)\right)_{\mu, \nu} \in \mathbb{C}<<z>>^{p \times q} \text { and } \\
R_{j}\left(z^{1 / m}\right) & \in \mathbb{C}<<z^{1 / m}>>^{p \times q}, \\
\sigma(R) & :=\max \left\{\sigma\left(R_{j}\right) ; R_{j} \neq 0\right\}=\max _{j, \mu, \nu} \sigma\left(R_{j \mu \nu}(z)\right)(\mathrm{cf} .(49)),
\end{aligned}
$$

acts on $w \in W\left(t_{0}\right)^{q}=\left(\mathbb{C}^{q}\right)^{t_{0}+\mathbb{N}}$ with $t_{0}>\sigma(R)^{m}$ via

$$
\begin{aligned}
(R \circ w)(t) & :=\sum_{j} R_{j}\left(t^{-1 / m}\right) w(t+j), t_{0}>\sigma(R)^{m}, \\
\mathcal{B}\left(R, t_{0}\right) & :=\left\{w \in W\left(t_{0}\right)^{q} ; R \circ w=0\right\} .
\end{aligned}
$$

In particular, a matrix $A\left(z^{1 / m}\right) \in \mathbf{P}^{n \times n}$ induces the state space equation and behaviors

$$
\begin{aligned}
x(t+1) & =A\left(t^{-1 / m}\right) x(t), t \geq t_{0}>\sigma(A)^{m}, \\
\mathcal{K}\left(A\left(z^{1 / m}\right), t_{0}\right) & :=\mathcal{B}\left(s \operatorname{id}_{q}-A\left(z^{1 / m}\right), t_{0}\right) \\
& =\left\{x \in W\left(t_{0}\right)^{n} ; x(t+1)=A\left(t^{-1 / m}\right) x(t)\right\} .
\end{aligned}
$$

Result 4.1 Since $\mathbf{P}$ satisfies the conditions of Remark 3.8 the notions and theorems of [4] also hold for $\mathbf{P}$ like for $\mathbb{C}(z)$ in [4] and for $\mathbf{K}=\mathbb{C}<<z>>$ in Sect. 3.

Corollary 4.2 Consider $A=A(z) \in \mathbb{C}<<z>>^{n \times n}$ and the state space system

$$
x(t+1)=A\left(t^{-1 / m}\right) x(t), t>\sigma(A)^{m} .
$$

(i) If $A(z)=A_{0}+A_{1} z+\cdots \in \mathbb{C}<z>^{n \times n}$ is a power series the system is e.s. if and only if all eigenvalues of $A_{0}$ have absolute value $<1$.

(ii) If $A(z)=z^{-k} B(z), B(z)=\sum_{i=0}^{\infty} B_{i} z^{i}, k>0$ and $B_{0}$ is not nilpotent then

$$
\exists t_{0}>\sigma(A)^{m} \forall t_{1} \geq t_{0}: \sup _{t \geq t_{1}}\left\|\Phi_{A\left(t^{-1 / m}\right)}\left(t, t_{1}\right)\right\|=\infty
$$

and the system is not e.s.

(iii) If for $A(z)$ as in (ii) the determinant of $\operatorname{det}(B(z))$ has the form $\operatorname{det}(B(z))=$ $b_{\ell} z^{\ell} c(z) \neq 0$ where $c(z)=1+\sum_{i=1}^{\infty} c_{i} z^{i}$ and $k n-\ell>0$ then (73) holds and the system is not e.s.

One can weaken the definition of exponential stability to weak exponential stability (w.e.s.) as in [5, Def. 2.4]. This is also preserved by behavior isomorphisms. For the state space system (72) w.e.s. holds if and only if

$$
\begin{aligned}
& \exists t_{0}>\sigma(A)^{m} \exists \rho=e^{-\alpha}<1(\alpha>0) \exists \mu>0 \exists \text { p.g. } \varphi \in \mathbb{C}^{t_{0}+\mathbb{N}}, \varphi>0, \\
& \forall t_{1} \geq t_{0}:\left\|\Phi_{A\left(t^{-1 / m}\right)}\left(t, t_{1}\right)\right\| \leq \varphi\left(t_{1}\right) \rho^{t^{\mu}-t_{1}^{\mu}}=\varphi\left(t_{1}\right) e^{-\alpha\left(t^{\mu}-t_{0}^{\mu}\right)} .
\end{aligned}
$$


The difference to e.s. is the exponent $\mu>0$. If $\mu<1$ the factor $\exp \left(-\alpha t^{\mu}\right)$ decreases more slowly than $\exp (-\alpha t)$. Corollary 4.2 obtains a slightly weaker form.

Corollary 4.3 Corollary 4.2 remains true for w.e.s. instead of e.s. with the following exception in part (i): If $A(z)=\sum_{i=0}^{\infty} A_{i} z^{i} \in \mathbb{C}<z>^{n \times n}$ then the system is e.s. and hence w.e.s. resp. unstable if $|\lambda|<1$ for all eigenvalues $\lambda$ of $A_{0}$ resp. $|\lambda|>1$ for at least one. If $|\lambda| \leq 1$ for all eigenvalues of $A_{0}$ and $|\lambda|=1$ for at least one the system may be w.e.s. in contrast to Corollary 4.2, (i), cf. Example 4.4.

Example 4.4 (Cf. [16, §6.1, §7.1]) Let $\alpha>0, \rho:=e^{-\alpha}, \mu=k_{0} / m, 0<k_{0}<m$. The signal $x(t):=e^{-\alpha t^{\mu}}=\rho^{t^{\mu}}$ satisfies

$$
\begin{aligned}
x(t+1) / x(t) & =\exp \left(-\alpha\left((t+1)^{\mu}-t^{\mu}\right)\right) \text {. But } \\
(t+1)^{\mu}-t^{\mu} & =t^{\mu}\left(\left(1+t^{-1}\right)^{\mu}-1\right)=\left(z^{-\mu}\left((1+z)^{\mu}-1\right)\right)\left(t^{-1}\right) \\
z^{-\mu}\left((1+z)^{\mu}-1\right) & =z^{-\mu} \sum_{k=1}^{\infty}\left(\begin{array}{l}
\mu \\
k
\end{array}\right) z^{k}=\sum_{k=1}^{\infty}\left(\begin{array}{l}
\mu \\
k
\end{array}\right)\left(z^{1 / m}\right)^{k m-k_{0}} \in \mathbb{C}<z^{1 / m}>.
\end{aligned}
$$

We define

$$
\begin{aligned}
a(z) & :=\exp \left(-\alpha \sum_{k=1}^{\infty}\left(\begin{array}{l}
\mu \\
k
\end{array}\right) z^{k m-k_{0}}\right)=1-\alpha \mu z^{m-k_{0}}+\cdots \in \mathbb{C}<z> \\
& \Longrightarrow a\left(z^{1 / m}\right)=\exp \left(-\alpha z^{-\mu}\left((1+z)^{\mu}-1\right)\right) \\
& =1-\alpha \mu z^{1-\mu}+\cdots \in \mathbb{C}<z^{1 / m}> \\
& \Longrightarrow a\left(t^{-1 / m}\right)=\exp \left(-\alpha\left((t+1)^{\mu}-t^{\mu}\right)\right), x(t+1)=a\left(t^{-1 / m}\right) x(t) \\
& \Longrightarrow x(t)=\exp \left(-\alpha\left(t^{\mu}-t_{0}^{\mu}\right)\right) x\left(t_{0}\right),|x(t)|=\exp \left(-\alpha\left(t^{\mu}-t_{0}^{\mu}\right)\right)\left|x\left(t_{0}\right)\right|
\end{aligned}
$$

This equation is w.e.s. with exponent $\mu$. But $a(0)=1$ and the equation is not e.s by Corollary 4.2 .

Acknowledgements Open access funding provided by University of Innsbruck and Medical University of Innsbruck.

Open Access This article is distributed under the terms of the Creative Commons Attribution 4.0 International License (http://creativecommons.org/licenses/by/4.0/), which permits unrestricted use, distribution, and reproduction in any medium, provided you give appropriate credit to the original author(s) and the source, provide a link to the Creative Commons license, and indicate if changes were made.

\section{References}

1. Anderson, B.D.O., Ilchmann, A., Wirth, F.R.: Stabilizability of linear time-varying systems. Syst. Control Lett. 62, 747-755 (2013)

2. Berger, T., Ilchmann, A., Wirth, F.R.: Zero dynamics and stabilization for analytic linear systems. Acta Appl. Math. 138, 17-57 (2015)

3. Bourlès, H., Marinescu, B.: Linear Time-Varying Systems. Springer, Berlin (2011)

4. Bourlès, H., Marinescu, B., Oberst, U.: Exponentially stable linear time-varying discrete behaviors. SIAM J. Control Optim. 53, 2725-2761 (2015) 
5. Bourlès, H., Marinescu, B., Oberst, U.: Weak exponential stability of linear time-varying differential behaviors. Linear Algebra Appl. 486, 1-49 (2015)

6. Chyzak, F., Quadrat, A., Robertz, D.: OreModules: a symbolic package for the study of multidimensional linear systems. In: Chiasson, J., Loiseau, J.-J. (eds.) Applications of Time-Delay Systems. Lecture Notes in Control and Information Sciences, vol. 352, pp. 233-264. Springer, Berlin (2007)

7. Hill, A.T., Ilchmann, A.: Exponential stability of time-varying linear systems. IMA J. Numer. Anal. 31, 865-885 (2011)

8. Hinrichsen, D., Pritchard, A.J.: Mathematical Systems Theory I. Springer, Berlin (2005)

9. McConnell, J.C., Robson, J.C.: Noncommutative Noetherian Rings. Graduate Studies in Mathematics, vol. 30. American Mathematical Society, Providence (2001).

10. Medina, R.: Stability analysis of nonautonomous difference systems with delaying arguments. J. Math. Anal. Appl. 335, 615-625 (2007)

11. Nowak, K.J.: Some elementary proofs of Puiseux's theorems. Acta Math. Univ. Iagell. 38, 279-282 (2000)

12. Oberst, U.: Stabilizing compensators for linear time-varying differential systems. Int. J. Control 89, 1-31 (2015). doi:10.1080/00207179.2015.1091949

13. Phat, V.N., Jeyakumar, V.: Stability, stabilization and duality for linear time-varying systems. Optimization 59, 447-460 (2010)

14. Robertz, D.: Recent progress in an algebraic analysis approach to linear systems. Multidimens. Syst. Signal Process. 26, 349-388 (2015). doi:10.1007/s11045-014-0280-9

15. Rugh, W.J.: Linear System Theory. Prentice Hall, Upper Saddle River (1996)

16. van der Put, M., Singer, M.F.: Galois Theory of Difference Equations. Springer, Berlin (1997) 